\title{
Reconstruction of partially occluded objects encoded in three-dimensional scenes by using digital holograms
}

\author{
Jonathan Maycock, Conor P. McElhinney, Bryan M. Hennelly, Thomas J. Naughton, \\ John B. McDonald, and Bahram Javidi
}

\begin{abstract}
We propose a task-specific digital holographic capture system for three-dimensional scenes, which can reduce the amount of data sent from the camera system to the receiver and can effectively reconstruct partially occluded objects. The system requires knowledge of the object of interest, but it does not require a priori knowledge of either the occlusion or the distance the object is from the camera. Subwindows of the camera-plane Fresnel field are digitally propagated to reveal different perspectives of the scene, and these are combined to overcome the unknown foreground occlusions. The nature of the occlusions and the effect of subwindows are analyzed thoroughly by using the Wigner distribution function. We demonstrate that a careful combination of reconstructions from subwindows can reveal features that are not apparent in a reconstruction from the whole hologram. We provide results by using optically captured digital holograms of real-world objects and simulated occlusions. (C) 2006 Optical Society of America

OCIS codes: $\quad 090.1760,100.6890,100.2000$.
\end{abstract}

\section{Introduction}

In this paper we explore the potential use of digital holograms in a three-dimensional (3D) scene reconstruction in which particular regions of interest are occluded under certain perspectives. The study of occlusions in digital holography has been actively researched of late. For the case of digital hologram watermarking, ${ }^{1}$ occlusions are considered attacks and occur in the hologram plane, resulting in a direct loss of data at that plane. The integration of computer graphics and holograms has also been investigated, necessitating the study of occlusion effects. ${ }^{2}$ Another technique, which attempts to overcome the problems of foreground occlusions in a complex scene, is integral imaging, which uses the concept of ray phase space to reconstruct the occluded images. It is based on ray optics and uses different view perspectives with a microlens array. ${ }^{3}$ This is a passive sensor, as opposed to active sensing as in digital holography.

J. Maycock (jmaycock@cs.nuim.ie), C. P. McElhinney, B. M. Hennelly, T. J. Naughton, and J. B. McDonald are with the Department of Computer Science, National University of Ireland, Maynooth, County Kildare, Ireland. B. Javidi is with the Department of Electrical and Computer Engineering, University of Connecticut, U-157, Storrs, Connecticut 06269.

Received 8 September 2005; revised 23 December 2005; accepted 23 December 2005.

0003-6935/06/132975- $11 \$ 15.00 / 0$

(C) 2006 Optical Society of America
We have simulated the addition of occlusions in free space ${ }^{4}$ (i.e., in the region between the object of interest and the camera), thus modeling opaque and nonopaque occlusions as they occur in nature. In our simulated experiments we use optically captured digital holograms of real-world objects. We show that by reconstructing the entire hologram it is possible to overcome the problems of foreground occlusions in a scene. This is achieved by propagating the wavefront to the calculated in-focus plane of the object of interest. However, for the purpose of this paper, we investigate finding a subset of pixels that can successfully reconstruct partially occluded background objects by selectively incorporating information from additional views of the scene. There are three reasons why this is useful. The first is that different windows in the hologram plane give different perspectives. In situations in which a nonopaque occlusion is present, the reconstruction plane may be corrupted by nonphysical light emanating backward from the occlusion. This light acts as noise and can saturate features on the object of interest. Careful selection of windows from the hologram plane reduces the influence of this noise and makes visible features that are not discernible when the whole hologram is used for reconstruction. The second benefit is that the computational complexity of hologram reconstruction is reduced if fewer pixels are used to reconstruct the scene. The third benefit is that reducing the number of pixels 


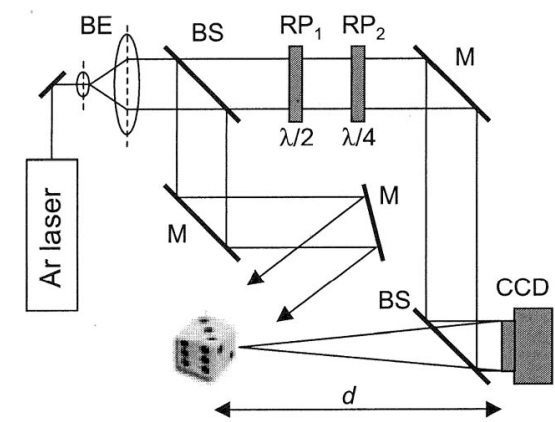

Fig. 1. Experimental setup for PSI: BE, beam expander; BS, beam splitter; RP, retardation plate; $\mathrm{M}$, mirror.

that must be sent over a communication channel will result in increased communication efficiency.

Holography ${ }^{5}$ is an established technique for recording and reconstructing real-world 3D objects. Digital holography ${ }^{6-11}$ has become feasible owing to recent advances in megapixel CCD sensors with high spatial resolution and high dynamic range. A technique known as phase-shift interferometry (PSI) was used to create our in-line digital holograms. ${ }^{12,13} \mathrm{~A}$ single hologram of a scene encodes multiple perspectives of the scene simultaneously, and we have used this property to reconstruct the scene from different viewpoints, thus overcoming occlusions. Digital holographic image processing is an area that has seen increased interest over the past few years. ${ }^{4,12-19}$

\section{Phase-Shift Digital Holography}

We record whole Fresnel fields with an optical system ${ }^{12,13}$ based on a Mach-Zehnder interferometer (see Fig. 1). A linearly polarized argon-ion (514.5 nm) laser beam is expanded and collimated and then divided into object and reference beams. The object beam illuminates a reference object placed at a distance of approximately $d=350 \mathrm{~mm}$ from a 10-bit $2028 \times 2044$ pixel Kodak Megaplus CCD camera. Let $U_{0}(x, y)$ be the complex amplitude distribution immediately in front of the $3 \mathrm{D}$ object. The reference beam passes through retardation plates $R_{1}$ and $R P_{2}$, and by selectively removing the plates we can achieve four phase-shift permutations, for each of which we record an interferogram. We use these four realvalued images to compute the camera-plane complex field $H_{0}(x, y)$ by PSI.8,10 We call this computed field a digital hologram.

A digital hologram $H_{0}(x, y)$ contains sufficient amplitude and phase information to reconstruct the complex field $U(x, y, z)$ in a plane at any distance $z$ from the camera. ${ }^{10-12}$ This can be calculated from the Fresnel approximation ${ }^{20}$ as

$$
\begin{aligned}
U(x, y, d)= & \frac{-i}{\lambda d} \exp \left(i \frac{2 \pi}{\lambda} d\right) \\
& \times H_{0}(x, y) * \exp \left[i \pi \frac{\left(x^{2}+y^{2}\right)}{\lambda d}\right],
\end{aligned}
$$

where $\lambda$ is the wavelength of the light and $*$ denotes a convolution. At $z=d$, and ignoring errors in digital propagation due to pixelation and rounding, the discrete reconstruction $U(x, y, z)$ closely approximates the physical continuous field $U_{0}(x, y)$.

As with conventional holography, a digital hologram encodes different views of a 3D object from a small range of angles. ${ }^{20,21}$ To reconstruct a particular two-dimensional (2D) perspective of the object, we must extract the appropriate windowed subset of pixels from the hologram and subject them to simulated Fresnel propagation. ${ }^{10-12}$ As the window explores the field, a different angle of view of the object can be reconstructed. The range of viewing angles is determined by the ratio of the window size to the full CCD sensor dimensions. Our CCD sensor has approximate dimensions of $18.5 \mathrm{~mm} \times 18.5 \mathrm{~mm}$, and so a $1024 \times$ 1024 pixel window has a maximum lateral shift of $9 \mathrm{~mm}$ across the face of the sensor. With an object positioned $d=350 \mathrm{~mm}$ from the camera, viewing angles in the range of $\pm 0.74^{\circ}$ are permitted. Smaller windows will permit a larger range of viewing angles at the expense of image resolution at each viewpoint.

\section{Wigner Distribution Function and Digital Holography}

\section{A. Wigner Distribution Function and Properties}

The WDF of a complex optical amplitude distribution provides a graphic means of simultaneously viewing a signal's spatial and spatial-frequency distributions and is particularly useful for visualizing localized signals. ${ }^{22-25} \Psi\{f(x)\}(x, k)$, which represents the WDF of a signal $f(x)$, is defined in terms of this spatial distribution in the following way:

$$
\begin{aligned}
\Psi\{f(x)\}(x, k)= & \int_{-\infty}^{\infty} f\left(x+\frac{\xi}{2}\right) f^{*}\left(x-\frac{\xi}{2}\right) \\
& \times \exp (-j 2 \pi k \xi) \mathrm{d} \xi
\end{aligned}
$$

where $k$ represents spatial frequency, (*) denotes complex conjugation, and $\Psi$ denotes the WDF operator. Often a plan-view outline of the signal's energy is used for illustration. Such a Wigner chart is shown in Fig. 2. The real-valued WDF has double the number of dimensions, i.e., a complex one-dimensional (1D) signal has a 2D WDF, while $2 \mathrm{D}$ signals have four-dimensional (4D) WDFs. The WDF has many properties ${ }^{26}$ :

(i) Shifting a signal in $x$ correspondingly shifts its WDF as

$$
\psi\{f(x-\xi)\}(x, k)=\psi\{f(x)\}(x-\xi, k) .
$$

(ii) The WDF has a convolution property, 


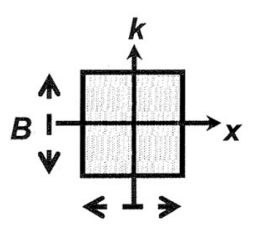

(a)

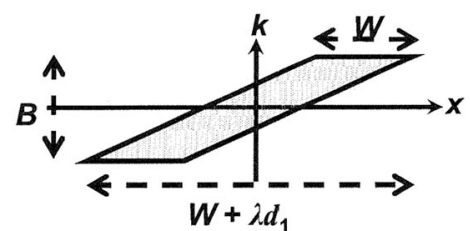

(b)

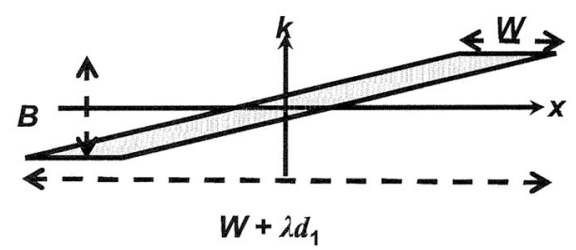

(c)

Fig. 2. Wigner chart of the propagating signal. (a) Wavefield immediately after the object, (b) Wigner chart after propagation of distance $d_{1}$, (c) Wigner chart after further propagation of distance $d_{2}$.

$$
\begin{aligned}
& \psi\left\{\int_{-\infty}^{\infty} f(\xi) g(x-\xi) \mathrm{d} \xi\right\}(x, k)=\int \psi\{f(x)\} \\
& \times\left(x-x^{\prime}, k\right) \psi\{g(x)\}\left(x^{\prime}, k\right) \mathrm{d} x^{\prime}, \\
& \psi\{f(x) g(x)\}(x, k)=\int_{-\infty}^{\infty} \psi\{f(x)\} \\
& \times\left(x, k-k^{\prime}\right) \psi\{g(x)\}\left(x, k^{\prime}\right) \mathrm{d} k^{\prime} .
\end{aligned}
$$

(iii) The WDF is bilinear. When two signals are added, the WDF of the sum is given by the sum of the individual WDFs plus an additional cross term,

$$
\begin{aligned}
& \psi\{f(x)+g(x)\}(x, k)=\psi\{f(x)\}(x, k)+\psi\{g(x)\}(x, k) \\
& +\int_{-\infty}^{\infty} 2 \Re\left[f(x-\xi / 2) g^{*}(x+\xi / 2)\right] \exp [-j 2 \pi k \xi] \mathrm{d} \xi .
\end{aligned}
$$

(iv) The projection of the WDF normal to the $k$ axis (i.e., integrating along $k$ ) gives the spatial intensity distribution of our signal, and the projection normal to the $x$ axis gives the frequency spectrum

$$
\begin{gathered}
|f(x)|^{2}=\int_{-\infty}^{\infty} \psi\{f(x)\}(x, k) \mathrm{d} k, \\
|F(k)|^{2}=\int_{-\infty}^{\infty} \psi\{f(x)\}(x, k) \mathrm{d} x .
\end{gathered}
$$

We note that it is possible for the energy of two signals that have been added together to overlap in the projection along $x$ and not to overlap in the projection along $k$ or vice versa. If the signals do not overlap in the projection that we are interested in, we may omit the cross term in Eq. (5) in all of the Wigner analyses.

(v) The Fresnel transform (FST), mentioned above, has the following effect on the WDF of a signal, $\psi\left\{F_{\lambda d}\{f(x)\}\left(x^{\prime}\right)\right\}\left(x^{\prime}, k^{\prime}\right)=\psi\{f(x)\}(x+\lambda d k, k)$. The effect of applying a FST to our signal can be expressed in terms of a matrix operating on the phase space coordinate vector

$$
\left[\begin{array}{l}
x^{\prime} \\
k^{\prime}
\end{array}\right]=\left[\begin{array}{cc}
1 & \lambda d \\
0 & 1
\end{array}\right]\left[\begin{array}{l}
x \\
k
\end{array}\right]
$$

This matrix is known as the $A B C D$ matrix or the ray-transfer matrix. The FST causes a shearing of a signal's WDF along the $x$ direction as shown in Figs. 2(b) and 2(c), in which we have shown the WDF for two Fresnel-transformed signals, $d 2>d 1$.

(vi) Signal localization. If the FT of a function is finite in extent, then the spatial distribution must have an infinite extent. This implies that since our band-limited signal exists over the entire extent of space, it must be sampled over all space. However, in many practical problems, it is assumed with a very large degree of accuracy that a signal is bounded within some finite region in both the spatial and the spatial-frequency domains. The spatial extent $W$ and the frequency extent $B$ are defined such that

$$
f(x) \approx 0 \quad|x|>W / 2, \quad \Im\{f(x)\}(k) \approx 0 \quad|k|>B / 2,
$$

and therefore the signal energy is negligible outside these regions.

(vii) Sampling. The WDF of a sampled signal has been investigated in detail by Stern and Javidi.27,28 Ideal sampling is accomplished by multiplying our signal by a train of Dirac delta functions or a comb function, $\delta_{T}(x)=\sum_{n=-\infty}^{\infty} \delta(x-n T)$, where $T$ is the sampling interval and $\delta(x)$ represents the Dirac delta function. This is equivalent to convolving the signal's WDF, $\psi\{f(x)\}(x, k)$, with $\psi\{\delta(x)\}(x, k)$, the WDF of the comb function. We note that

$$
\psi\left\{\delta_{T}(x)\right\}(x, k)=\frac{1}{2 T} \sum_{n} \sum_{q}(-1)^{q n} \delta\left(x-\frac{n T}{2}\right) \delta\left(x-\frac{q}{2 T}\right) .
$$

(viii) Ray tracing, the WDF, and the corner coordinate matrix (CCM). The WDF offers a unified interpretation of optics-wave, geometric, and quantum. Ray tracing is a widely used application of geometrical optics and involves following the paths of light rays as they pass through an optical system. For a specific ray with known position $(x)$ and direction (spatial frequency $k$ ), we may map any change in position and direction due to an optical element, including free space, by using the ray-transfer matrix for that optical element. We have shown above, in the case of free-space propagation in the paraxial approximation, that the ray-transfer matrix may also be used to map changes in the WDF of a signal. This has led to the development of the CCM, ${ }^{29}$ a $2 \mathrm{D}$ array containing a series of $x-k$ coordinates that define the 
boundary of a localized signal in phase space. For example, for the signal in Fig. 2(a), we will have a $\mathrm{CCM}$ of

$$
\mathbf{S}=\left[\begin{array}{rrrr}
-W / 2 & W / 2 & W / 2 & -W / 2 \\
B / 2 & B / 2 & -B / 2 & -B / 2
\end{array}\right] .
$$

After Fresnel transformation for a distance $d$ [e.g., the signals shown in Figs. 2(b) and 2(c)], the new corner coordinates are given by

$$
\mathbf{S}^{\prime}=\left[\begin{array}{cc}
1 & \lambda d \\
0 & 1
\end{array}\right] \mathbf{S}
$$

These ideas have led to an optimization of all FST algorithms. ${ }^{29}$ We note that Eqs. (10) and (11) may easily be extended for an arbitrary number of coordinates. ${ }^{30}$

(ix) The WDF of a rectangular function. We define the rect function as

$$
\begin{aligned}
\operatorname{rect}(x / w) & =1 & \forall|x|<w / 2 \\
& =0 & \forall|x|>w / 2 .
\end{aligned}
$$

The WDF of $\operatorname{rect}(x / w)$ has been investigated in the context of diffraction ${ }^{31}$ from a thin slit and is given by

$$
\psi\{\operatorname{rect}(x / w)\}(x, k)=\frac{\sin (k[-2|x|+w])}{k},|x|<w / 2,
$$

which is a type of sinc function in $k$ for the range of $x$ values allowed. The scale of this sinc function, $p(x, w)$, is a function of both the width of the rect function and the value of $x$. For values of $x$ that are much less than $w$, the sinc function will be narrow. As $x$ approaches the boundary, the sinc function broadens in $k$. This effect is due to diffraction at the boundaries. In the following analysis we will assume that $w \gg \lambda$ and that we may therefore ignore the effect of diffraction at the edges. In this case we may write

$$
\psi\{\operatorname{rect}(x / w)\}(x, k)=\delta(k), \quad|x|<w / 2 .
$$

We note that this approximation conflicts with the existence conditions of the WDF (Ref. 23), but it will simplify the proceeding analysis considerably while retaining a high degree of accuracy.

(xi) The WDF of a rectangular occlusion. The transmission function of a rectangular occlusion of width $w$ may be written as

$$
\operatorname{occlusion}(x)=1-\operatorname{rect}(x / w) .
$$

Using Eqs. (5) and (15), we may calculate the WDF of the occlusion to be

$$
\psi\{\operatorname{occlusion}(x)\}(x, k)=\psi\{1-\operatorname{rect}(x / w)\}(x, k)
$$

$$
\begin{aligned}
= & \delta(k)-\psi\{\operatorname{rect}(x / w)\}(x, k) \\
& -\cos (\pi x k) \operatorname{sinc}(w k) .
\end{aligned}
$$

Once again, if $w \gg \lambda$, we may ignore the effect of diffraction at the edges and use the following approximation for ease of analysis:

$$
\psi\{\operatorname{occlusion}(x)\}(x, k)=\delta(k), \quad|x|>w / 2 .
$$

We are now in a position to discuss digital holography from the perspective of Wigner and to analyze (i) the effect of taking different windows within our hologram for reconstruction and (ii) the effect of occlusions on a digital holography system.

\section{B. Wigner Distribution Function and Digital Holography}

The experimental results displayed in this paper are obtained by using phase-shifting digital holography. In the following analysis we consider (without any loss of generality) such a system to be summarized by a single camera capable of measuring the complex amplitudes at the individual pixel positions. The wave field emanating from an object in the plane just after the object (plane of focus) is denoted $O(x)$ and has a WDF denoted by $\psi\{O(x)\}(x, k)$. The Wigner chart for the signal will be of the form shown in Fig. 2(a). After Fresnel propagation, the signal's chart now takes the form shown in Fig. 2(b), and the WDF of the propagated signal is given by $\psi\{O(x)\}$ $(x+\lambda d k, k)$. Capture by the CCD is equivalent to multiplying by a comb function (sampling interval equal to $T$, the distance between the pixels), which has been convolved by a rect function with a width equal to that of a CCD pixel, $w_{p}$. This is followed by multiplying by a rect function that is representative of the size $\left(w_{c}\right)$ and position $\left(x_{0}\right)$ of the CCD aperture. Thus the CCD function is given by

$$
\operatorname{CCD}\left(x-x_{c}\right)=\left[\delta_{T}(x) * \operatorname{rect}\left(\frac{x}{w_{p}}\right)\right] \times \operatorname{rect}\left(\frac{x-x_{c}}{w_{c}}\right) .
$$

Using Eqs. (3), (4a), (4b), (13), and (14), we may calculate the WDF of the $\operatorname{CCD}(x)$ in Eq. (18) as

$$
\begin{aligned}
& {\left[\psi\left\{\delta_{T}(x)\right\}(x, k) *^{x} \psi\{\right.}\left.\left.\operatorname{rect}\left(x / w_{p}\right)\right\}(x, k)\right] \\
& *^{k} \psi\left\{\left\{\operatorname{rect}\left(x / w_{c}\right)\right\}\left(x-x_{c}, k\right),\right.
\end{aligned}
$$

where the superscript on the asterisk denotes the coordinate vector along which the $1 \mathrm{D}$ convolution takes place. Capturing the digital hologram is equivalent to multiplying our complex signal by $\operatorname{CCD}(x)$. The WDF is therefore equivalent to a convolution of the WDFs along the $k$ axis:

$$
\psi\{O(x)\}(x-\lambda d k) *^{k} \psi\{\operatorname{CCD}(x)\}(x, k) .
$$

Recently Wigner analysis has found a significant application in digital holography. The generalized sam- 


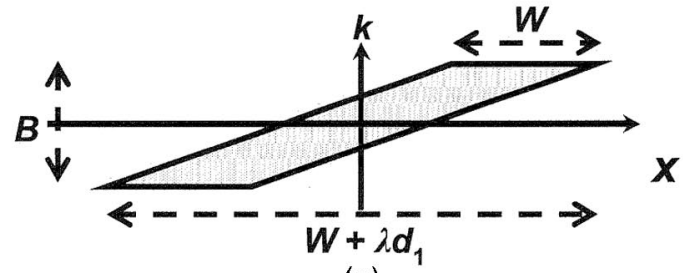

(a)

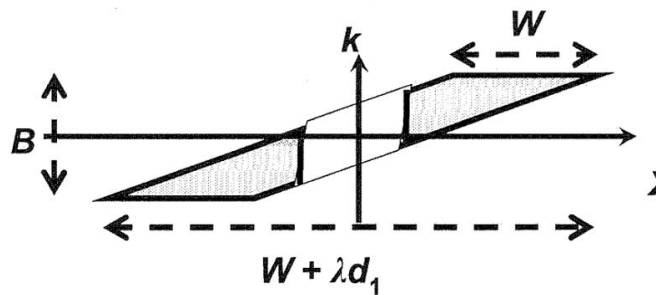

(c)

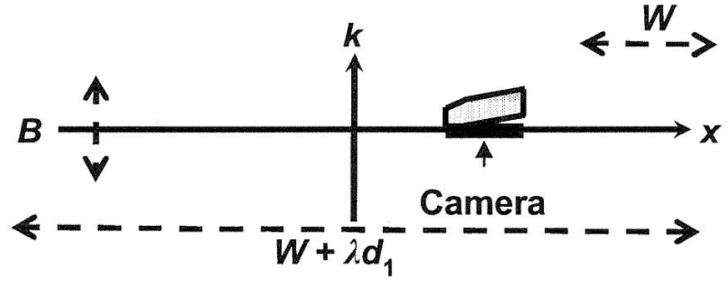

(e)

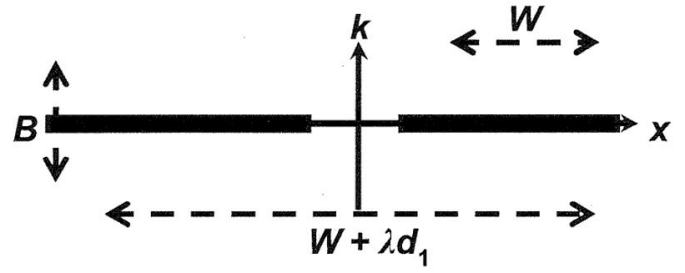

(b)

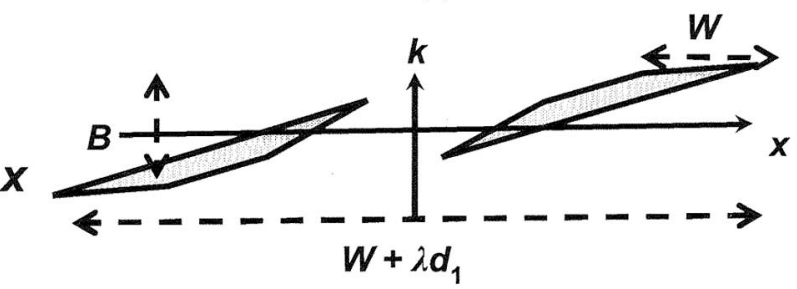

(d)

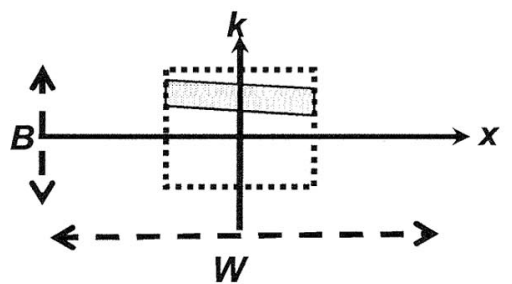

(f)

Fig. 3. (a) Wigner chart of the object signal after propagating a distance $d_{1}$ to the occlusion, (b) occlusion Wigner chart, (c) Wigner chart of the occluded wavefront obtained by convolving (a) and (b), (d) Wigner chart after the occluded wave field propagation of a distance $d_{2}$ to the CCD plane, (e) Wigner chart of the CCD and the captured wave field, (f) Wigner chart of the reconstructed signal against the total reconstructed signal.

pling theorem, ${ }^{27,28}$ which can be derived from Eqs. (9) and (20) above, allows us to improve the capacity of any digital holographic system. ${ }^{32}$ Simply stated, we need only ensure that the CCD bandwidth is greater than the local bandwidth of the incident signal, even if this local bandwidth lies far outside the bandwidth of the CCD. From this point on, we will assume that the optical recording is set up to ensure that no aliasing occurs in the process and that the sampling is ideal (i.e., the pixels are ideal delta functions). Thus we will entirely ignore the effect of sampling. Numerical implementations of the FSTs will also be assumed to be ideal. The true effect of CCD sampling and numerical Fresnel transformations may be found in great detail, ${ }^{33}$ but these effects have little influence on the results presented in this paper. As such, recording is summarized as convolving the signal's WDF with Eq. (14). The position of the WDF of the rect function can shift in $x$ depending on the center of the CCD (hologram) window. In Subsection 3.C we discuss opaque occlusions and nonopaque occlusions by using the WDF. In the latter case we show how astute positioning of the camera (or equivalently a choice of some subsection of the CCD recording) can lead to a lower signal-to-noise ratio in numerical object reconstruction.

\section{Occluded Objects and the Wigner Distribution} Function

We consider first the case of an opaque planar occlusion of width $w_{o}$, positioned at a distance of $d_{1}$ from the object and of $d_{2}$ from the CCD. The plane of the occlusion is normal to the axis of propagation. The occlusion may be modeled with the aid of a rect function as shown in Eq. (14). We may change the lateral position of the occlusion by setting $x \rightarrow x-x_{o}$ in Eq. (14), where $x_{0}$ is the position of the center.

The object wave field has the Wigner chart shown in Fig. 2(a). The object has a width $W$, and at each position over this width we assume rays of light to be traveling in all directions over a range of angle proportional to the bandwidth $B$. The power of the light in a given position or direction is represented by the height of the WDF at that coordinate. Since we are considering the general case, we may use the plan view of a square WDF for illustrative purposes, where $O(x)$, with WDF $\psi\{O(x)\}(x, k)$, propagates a distance $d_{1}$. The CCM, defining the shape, is given by Eq. (10). After propagation, the WDF becomes $\psi\{O(x)\}\left(x+\lambda d_{1} k, k\right)$. The resulting Wigner chart is shown in Fig. 3(a), and the new CCM is defined by Eq. (11), where we set $d=d_{1}$. In this plane the wave field is multiplied by occlusion $\left(x-x_{0}\right) / w_{0}$. The WDF of the occlusion, $\psi\left\{\right.$ occlusion $\left.\left(x / w_{o}\right)\right\}(x, k)$, is defined by an approximation in Eq. (16) and is illustrated in Fig. 3(b). Using Eqs. (3) and (4), we may define the WDF of the wave field immediately after the occlusion as

$$
\psi\{O(x)\}\left(x^{\prime}, k\right) *^{k} \psi\left\{\operatorname{rect}\left(\text { occlusion } x^{\prime} / w_{o}\right)\right\}\left(x^{\prime}-x_{o}, k\right),
$$




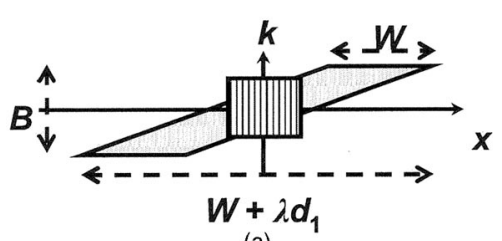

(a)

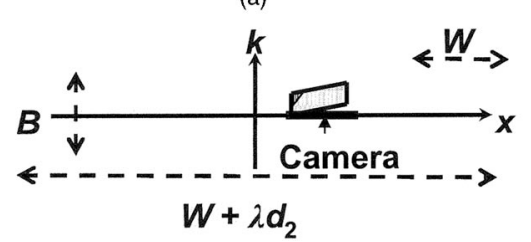

(c)

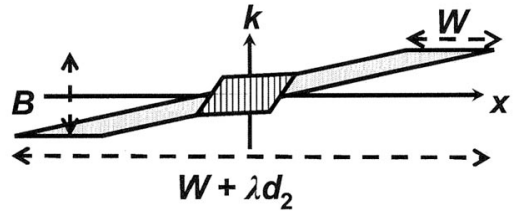

(b)

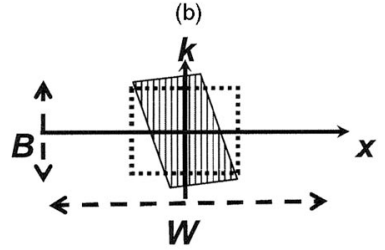

(d)

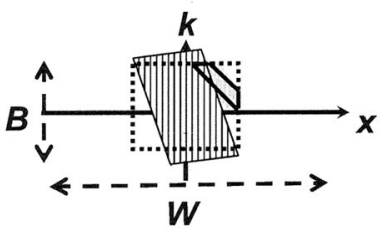

(e)
Fig. 4. (a) Wigner chart of both the occluded wave field (having already propagated a distance $d_{1}$ ) and the second wave field, (b) Two Wigner charts after propagation to the CCD plane, (c) Wigner charts after propagation to the CCD and the result of convolution with the signal's WDF, (d) Wigner chart of the reconstructed signals in the plane of the first object if the CCD had been large enough to capture the entire wave fields during recording, (e) reconstruction using the signal in (c) plotted against total reconstruction. where $x^{\prime}=x+\lambda d$. Taking the definition of the WDF of the occlusion given in Eq. (16), we illustrate the new Wigner chart in Fig. 3(c). We now require two CCMs. The coordinates for these two CCMs can be easily derived by using geometry on the original CCM.

The signal now propagates a distance $d_{2}$, and there is further shearing along the $x$ axis. The Wigner chart is shown in Fig. 3(d). The two new CCMs may be calculated by finding the product of the old CCMs with the Fresnel matrix in Eq. (11). The WDF of the signal at this point is

$$
\psi\{O(x)\}\left(x^{\prime \prime}, k\right) *^{k} \psi\left\{\operatorname{rect}\left(\text { occlusion } x^{\prime} / w_{o}\right)\right\}\left(x^{\prime \prime}-x_{o}, k\right),
$$

where $x^{\prime \prime}=x+\lambda\left(d_{1}+d_{2}\right) k$. The signal is then multiplied by $\operatorname{CCD}\left(x-x_{c}\right)$, the CCD transmission function. Based on previous discussions concerning CCD sampling, it is sufficient for the present analysis to assume ideal sampling and interpolation and to describe the CCD by using only $\operatorname{CCD}(x)=\operatorname{rect}\left(x / w_{c}\right)$. We show the WDF of such a signal in Fig. 3(e) with the approximation that it is a delta function over some region of space as in Eq. (5). In this figure we also show the section of the signal that would be cut out by this finite delta function after convolution along $k$. Once again, the new CCM may be found by applying geometry in an obvious fashion to the old CCMs. The WDF of the signal after capture by the $\mathrm{CCD}$ is given by

$$
\begin{aligned}
\psi\left\{\operatorname{hol}\left(x^{\prime \prime}\right)\right\} & \left(x^{\prime \prime}, k\right)=\left[\psi\{O(x)\}\left(x^{\prime \prime}, k\right) *^{k}\right. \\
\times \psi & \left.\left\{\operatorname{rect}\left(\operatorname{occlusion} x^{\prime} / w_{o}\right)\right\}\left(x^{\prime \prime}-x_{o}, k\right)\right] *^{k} \\
& \times \psi\left\{\operatorname{rect}\left(\operatorname{rect} x^{\prime \prime} / w_{o}\right)\right\} \times\left(x^{\prime \prime}-x_{c}, k\right) .
\end{aligned}
$$

A numerical FST of distance $-\left(d_{1}+d_{2}\right)$ is then applied to the signal. Assuming the numerical transform to be ideal, we can give the reconstructed signal by $\psi\left\{\operatorname{hol}\left(x^{\prime \prime}\right)\right\}\left(x-\lambda\left(d_{1}+d_{2}\right), k\right)$. The intensity can be found by using Eq. (5). The Wigner chart for this signal is illustrated in Fig. 3(f), plotted against the backdrop of the original Wigner chart. We have recovered a certain amount of the signal's energy in phase space. We may interpret this in one sense as having recovered a specific range of angles for different positions. As we vary the position of the camera $x_{c}$ and its width $w_{c}$, we may vary the range of angles recovered for each $x$. In certain cases the recovered angles will not exist because they have been removed by the occlusion. The effect of the occlusion will vary depending on the position and width of the camera. We note that while we have made large approximations in our illustrations, Eq. (23) is free of these approximations if we use the correct definitions of the WDF of a rect function.

When we consider the case of a nonopaque occlusion, we immediately find an application of the theory outlined above. In this case we have the sum of two signals to deal with; as before, we have the occluded wave field a distance $d_{2}$ from the CCD, and we have a second signal that lies within the occlusion. If these two complex signals are recorded and an inverse Fresnel transformation is used to return to the in-focus plane of the first object, we find that nonphysical light from the second object will be present as a source of noise. Not only will the intensity of this second signal be a source of noise but so will its interference with the desired wave field. We now demonstrate how to minimize the signal-to-noise ratio for the desired signal by making an appropriate choice of CCD position.

The bilinear property (v) above implies that the WDF of the sum will be given by the sum of the WDFs plus some cross term. From property (xi), we know that for a given projection the cross term will exist only in the region of overlap of the signals for that projection. We will include the cross term in our analysis, but we will ignore it in the illustrations of the Wigner charts given in Fig. 4. We show the Wigner charts of the two 


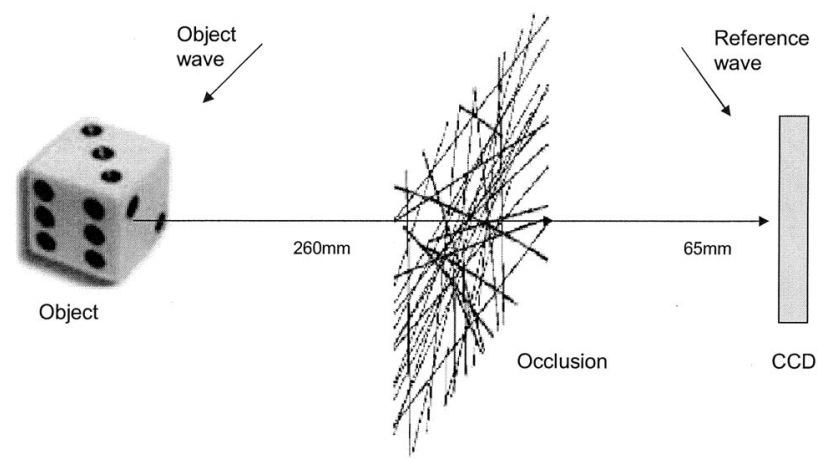

Fig. 5. Simulated experimental setup with an occlusion positioned at $260 \mathrm{~mm}$ from the object.

signals in Fig. 4(a). We have the occluded WDF as before, and now we also have a second wave field. At this point we have three CCMs: two describing the first signal and one for the second. After propagating a distance $d_{2}$, both signals are sheared along the $x$ axis, and the new CCMs may be easily calculated as before. The Wigner charts of the sheared wave fields are illustrated in Fig. 4(b). A CCD then captures the wave field in some region, and the resulting Wigner chart is shown in Fig. 4(c). The new CCMs can be calculated geometrically. Had the entire wave field been captured and an inverse numerical FST applied to return to the plane of the first object, the Wigner chart would look like that shown in Fig. 4(d). In this case we have recovered all the information that we can for the first object, but it overlaps with the second signal and is saturated with the second signal's intensity and their common interference. In Fig. 4(e) we show the Wigner chart for the CCD hologram that was inverse Fresnel transformed. We can see that while we have less of our signal's energy, we have an even smaller amount of the corrupting signals' energy. Thus, by changing our CCD position (or equivalently the region of the recorded hologram that we use in our reconstruction), we may recover multiple projections, each with a higher signal-to-noise ratio for a given region and over a different angle of view. We demonstrate these results by using experimental data in the following sections. It is straightforward to find the expression for the WDF of the recorded hologram and of the reconstructed wave field.

\section{Reconstruction of an Occluded Object with Digital Holography}

Any complex scene can contain foreground objects that occlude one's view of background objects of interest. Digital holography provides a novel way to overcome these occlusions by permitting unobstructed reconstructions of desired objects to be computed. ${ }^{4}$ The $3 \mathrm{D}$ objects used in our simulations were reconstructed from whole Fresnel fields of two objects: a die and a bolt. They were captured by using the phase-shift digital holography apparatus described in Section 2. Each Fresnel field is represented as a $2048 \times 2048$ complex-valued matrix.

To demonstrate the capability of digital holography to overcome problems of occlusion, we simulated the addition of a nonopaque occlusion to a scene containing a die object. The simulated experimental setup is shown in Fig. 5. This is the part of the optical apparatus from Fig. 1 between the object and the camera. It depicts a situation in which a foreground occlusion is obstructing the view of a background object of interest. Each pixel in the occlusion has a phase value chosen with uniform probability from the range $[0,2 \pi)$, which simulates a diffuse reflective object. This occlusion was positioned $260 \mathrm{~mm}$ from the object. The object was placed $325 \mathrm{~mm}$ from the camera (hologram plane). The hologram plane describes a 2D complex-valued signal representing a coherent wavefront that was incident on, and reflected from, a diffuse 3D object. By applying a discrete version of Eq. (1) to this complex wavefront, we can generate the whole Fresnel field in any plane. Simulated Fresnel propagation by a distance $z=-d$ to the camera plane gives us the whole Fresnel field that would be generated through PSI by the given object.

In this simulation we demonstrate how digital holograms, through Fresnel propagation to the correct distance, have the capability of overcoming the problems of foreground occlusions, which can be present in a scene. In Fig. 6(a) both the object and the occlusion are in focus simultaneously, as would be the case under the weak-perspective model. Figure 6(b) shows the object wavefront $260 \mathrm{~mm}$ from the camera, where the opaque occlusion was positioned. In Fig. 6(c) we see the reconstruction of the object by using the full hologram. Although the quality of this reconstruction is not equivalent to a reconstruction of the unoc-

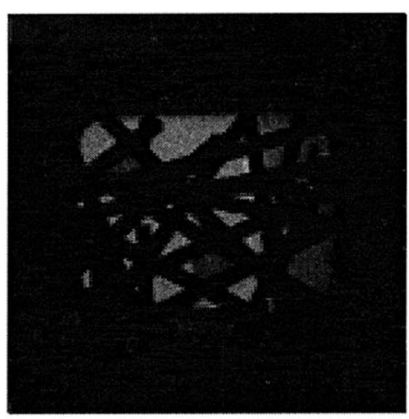

(a)

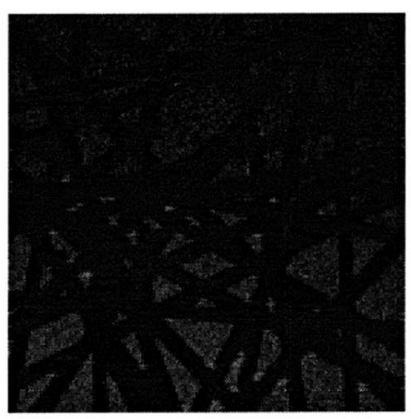

(b)

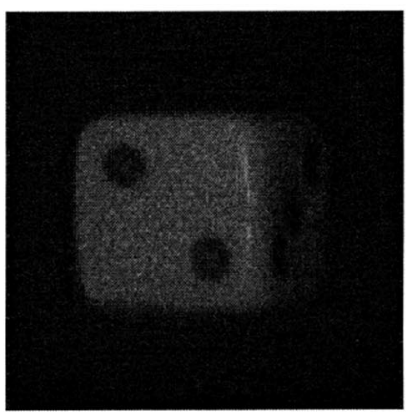

(c)
Fig. 6. Occlusion positioned at $280 \mathrm{~mm}$ from 3D object: (a) a weak perspective view of the scene, (b) the occlusion plane, (c) the reconstruction along the optical axis of the die. 


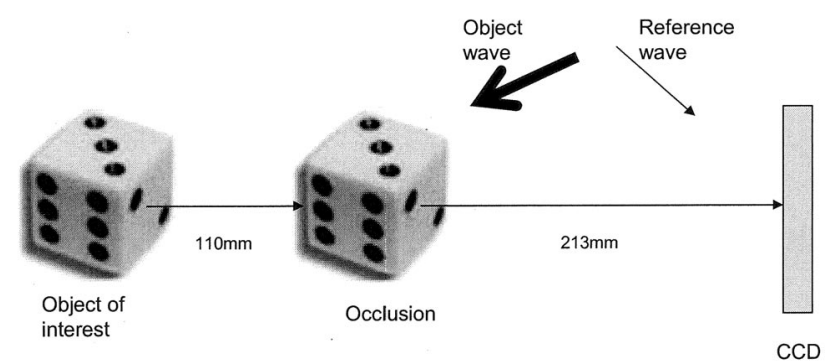

Fig. 7. Simulated experimental setup in which one die acts as an occlusion to a second die.

cluded die, object information that is not visible owing to the occlusion in the weak-perspective model becomes visible through the use of digital holography and Fresnel propagation to the object plane.

We will now investigate task-specific scene reconstruction by using a subsection of the hologram. The task we are interested in is one of trying to reveal a hidden feature on an occluded object, and we achieve this by taking a subset of pixels (a window) from the hologram and reconstructing from that perspective. By taking a smaller window from the hologram plane, we can reduce the effects of the out-of-focus occluding object on the background object of interest, and thus it is possible to reveal features that had been saturated by noise and therefore not visible. To verify this phenomena, we deal with a situation by using experimentally recorded PSI data. We have two object wave fields at two different distances from the hologram plane. The wave field from the background object has been occluded by the presence of the foreground object (see Fig 7). When propagating to the reconstruction plane of the background object, nonphysical light from the foreground object saturates this reconstruction. In Fig. 8(a) the entire hologram is used to reconstruct the object of interest. However, the three dots that are on the right-hand side of the die have been completely saturated by the out-of-focus occluding object and cannot be discerned. When the top right-hand corner $(1024 \times 1024$ pixels compared with the available $2048 \times 2048$ pixels) of

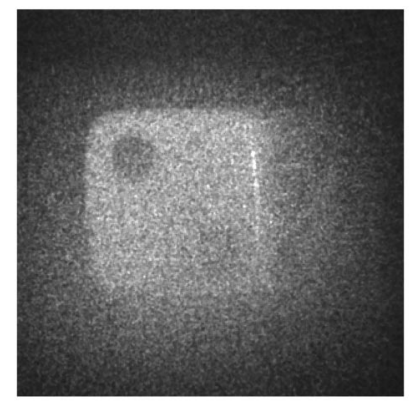

(a)

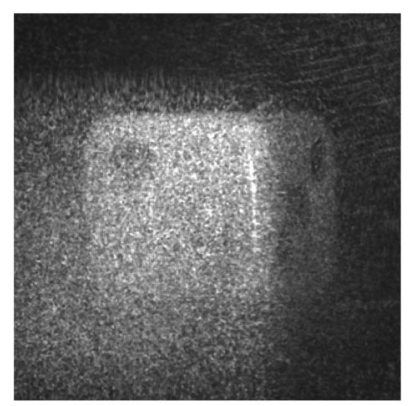

(b)
Fig. 8. Advantage of using a smaller window of pixels over the entire set of pixels when an occluded object is reconstructed: (a) reconstruction using the entire set of pixels and (b) reconstruction of the scene using the top right $1024 \times 1024$ window of pixels. the hologram is used to reconstruct the object, as is shown in Fig. 8(b), at least two of the dots come into view. It should be noted that median filtering with a neighborhood of $7 \times 7$ pixels was applied to both reconstructions.

We argue that the capability of revealing hidden features of a partially occluded object is one of the benefits of using smaller windows in the hologram plane for reconstruction. It should be noted that this is relevant only if a nonopaque occlusion is present. This is due to the fact that the out-of-focus light from the foreground occlusion can saturate background object features. In the case of an opaque occlusion, smaller windows will not have any benefit over using the entire hologram in revealing object features.

\section{Automated Task-Specific System to Reconstruct Occluded Background Objects}

Our proposed automated task-specific system attempts to reconstruct occluded objects by using a subset of available pixels. The task here is to find a subset of pixels that can produce reconstructions of the object that are visually close to what the entire set of pixels would give. As was discussed in Subsection 4.A, using smaller windows makes it possible to reveal object features that cannot be discerned when the entire hologram is used and when a nonopaque occlusion is present in the scene. There are two more reasons why using smaller windows of pixels to reconstruct an occluded object should be considered. The first is that the computational complexity of hologram reconstruction is reduced if fewer pixels are used to reconstruct the scene. A fast Fourier transform, which has complexity $n \log _{2} n$, is used to numerically reconstruct the hologram. By splitting the entire set of pixels into four windows and reconstructing them separately, the complexity is reduced to $n \log _{2}(n / 4)$. Of course, by choosing fewer pixels than are available, the computational complexity is reduced by a more significant factor. Another benefit of using subsections of the hologram under reconstruction is that reducing the numbers of pixels needed to reconstruct a scene will result in an increased communication efficiency during transmission over a communication channel.

The difference between our proposed system and a traditional holographic system $4,12,13$ is shown in Fig. 9. Once again, we have used PSI to capture our holograms, which meant the loop was closed between the Fresnel field and the output. However, in a holographic single-exposure on-line setup, ${ }^{34}$ the loop would be closed between the CCD and the output. In our system the output is not the complete Fresnel field obtained by the CCD, but rather a subset of pixels that can be used to reconstruct the occluded object.

We have developed a general algorithm that automates the process of choosing a subset of pixels to reconstruct an occluded object. It is parameterized to output five $512 \times 512$ possibly overlapping windows. Our algorithm needs no a priori knowledge of either the position of the object or the type of occlusion present in the scene. To calculate the position at which to reconstruct the scene, we use a depth-from- 


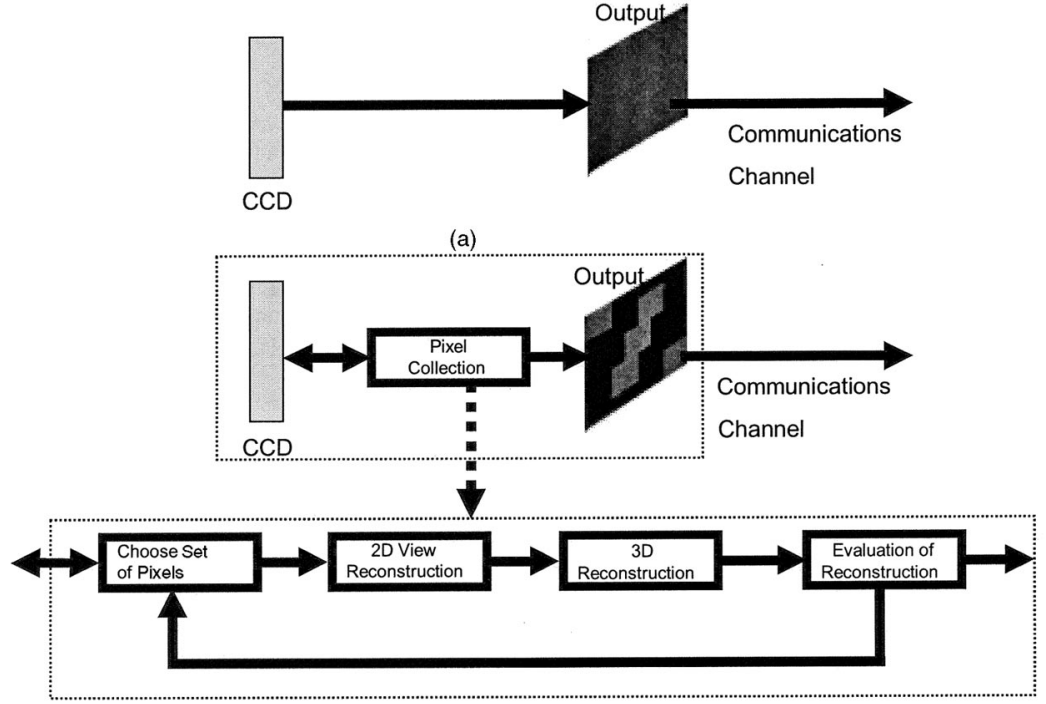

(b)
Fig. 9. Comparison between (a) a traditional holographic system and (b) the proposed automated task-specific holographic system. defocus technique. ${ }^{35}$ This technique calculates the depth at which the majority of information about the object of interest is in focus. Our digital hologram is split into $256 \times 256$ sections, giving us an $8 \times 8$ grid of windows (see Fig. 10). We choose a starting window for our search by locating the $512 \times 512$ window centered around the pixel with the highest intensity. A search is then sequentially carried out along each of the eight directional vectors from the starting window (see Fig. 10). In each step we shift the window by 256 pixels along the current direction. We reconstruct each window and combine each one with the reconstruction of the starting window. This is accomplished by taking the pixel with the highest intensity from each of the two reconstructions. To evaluate this combination, we compared it with a reconstruction of the unoccluded object by using an image-quality metric. We choose the normalized rms (NRMS) defined as

$$
\begin{aligned}
D\left(U^{\prime}\right)= & \left(\sum_{m=0}^{N_{x}-1} \sum_{n=0}^{N_{y}-1}\left[\left|U_{0}(m, n)\right|^{2}-\left|U^{\prime}(m, n)\right|^{2}\right]^{2}\right. \\
& \left.\times\left\{\sum_{m=0}^{N_{x}-1} \sum_{n=0}^{N_{y}-1}\left[\left|U_{0}(m, n)\right|^{2}\right]^{2}\right\}^{-1}\right)^{1 / 2},
\end{aligned}
$$

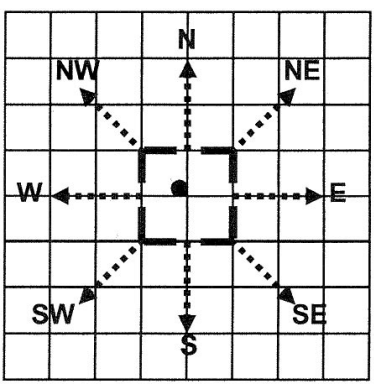

Fig. 10. Illustration of the grid structure, the starting window containing the highest intensity pixel, and the directional vectors used by the algorithm. where $U$ is the reconstruction of the unoccluded object, $U^{\prime}$ is the combined reconstruction, $(m, n)$ are discrete spatial coordinates in the reconstruction plane, and $N_{y}$ and $N_{x}$ are the height and width of the reconstructions, respectively. We store the window along each directional vector, which results in the lowest reconstruction error value. This gives us a possible total of eight new windows plus the starting window. From this set, we choose the starting window and the subset of four windows that resulted in the lowest error values.

Our algorithm is generalized to work with both opaque and nonopaque occlusions. However, a scene containing an opaque occlusion was simulated to test our algorithm. This meant that we lost the benefit of possibly revealing hidden features on the partially occluded object by reconstructing it with smaller windows from the hologram plane. However, we argue that even in the case of an opaque occlusion, the benefits of reducing the computational complexity and of using fewer pixels for reconstruction warrant investigation into the calculation of a subset of pixels. The setup for this experiment is shown in Fig. 11. It depicts a situation in which the propagated wave-

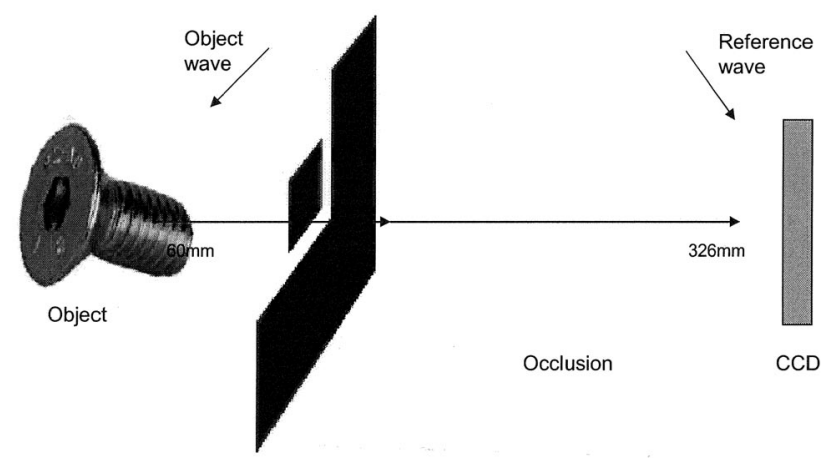

Fig. 11. Simulated experimental setup with an opaque occlusion positioned $60 \mathrm{~mm}$ from the object. 


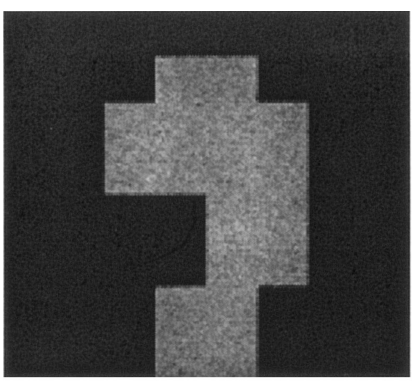

(a)

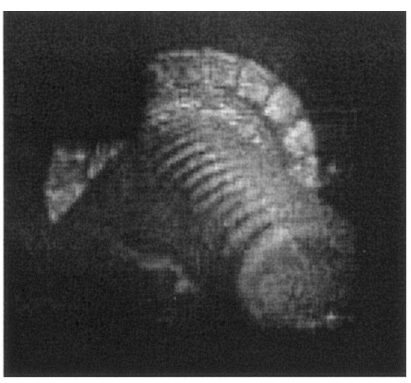

(b)

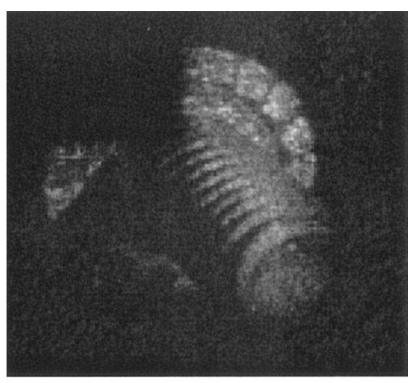

(c)
Fig. 12. Reconstruction of the scene using (a) the pixels selected by the algorithm, (b) the entire set of pixels, (c) the combination of five $512 \times 512$ pixel region reconstructions. front of the object was severely occluded by an opaque surface. At the occlusion plane, $72 \%$ of the propagated object wavefront was blocked. The pixels chosen by our automated system are shown in Fig. 12(a). Each pixel in the final reconstruction is the maximum amplitude value from the corresponding pixels in the partial reconstructions. The reasoning here is that in this situation there is no nonphysical light generated by the occlusion, and thus intensity values should relate to object information. In the case of a nonopaque occlusion the complex-valued pixels from different subwindows should be added together. Figure 12(c) shows the reconstruction obtained by combining the five windows chosen by our automated system. The reconstruction using the entire set of available pixels is shown in Fig. 12(b) for comparison. Our system produced a NRMS error of 0.82817 , whereas the reconstruction using the entire set of available pixels produced an error of 0.71302. Our system achieved a reconstruction quality that deviated only by approximately $12 \%$ from the reconstruction obtained by using the total signal energy. It should be noted that the effect of the occlusion is such that even when the entire set of pixels is used, parts of the bolt are still not visible.

This implementation of the algorithm uses the NRMS to determine the subset of pixels output by the system. However, if the task was one of object recognition, our algorithm facilitates the integration of an object-recognition function in place of the NRMS. In this case a subset of pixels would be returned if the object were recognized.

\section{Conclusion}

The nature of opaque and nonopaque occlusions and the effect of reconstructing subwindows from the hologram plane have been analyzed thoroughly by using the WDF. Furthermore, we have shown that a subset of pixels can be used for $3 \mathrm{D}$ reconstruction of complex scenes. In the presence of a nonopaque occlusion, this can have the benefit of revealing hidden features on a partially occluded object. Independent of the type of occlusion, using a subset of pixels has the advantage of being computationally less complex when the hologram is reconstructed and results in greater communication efficiency during transmission over a communication channel. We have detailed a proposed automated task-specific holographic sys- tem, which has the capability of reconstructing partially occluded objects and of reducing the amount of data sent from the camera system to the receiver. This system would be useful in situations in which transmission of the whole digital hologram, or exhaustive reconstruction of every perspective, was not feasible.

The authors thank Enrique Tajahuerce and Yann Frauel for use of their digital hologram data. This paper has emanated from research conducted with the financial support of the Science Foundation Ireland, Enterprise Ireland, and the Embark Initiative of the Irish Research Council for Science, Engineering, and Technology.

\section{References}

1. H. Kim and Y. H. Lee, "Optimal watermarking of digital hologram of 3-D object," Opt. Express 13, 2881-2886 (2005).

2. O. Bimber, T. Zeidler, A. Grundhfer, G. Wetzstein, M. Mhring, S. Kndel, and U. Hahne, "Interacting with augmented holograms," in Practical Holography XIX: Materials and Applications, T. H. Jeong and H. I. Bjelkhagen, eds., Proc. SPIE 5742, 41-54 (2005).

3. S.-H. Hong and B. Javidi, "Three-dimensional visualization of partially occluded objects using integral imaging," IEEE J. Display Technol. 1, 354-359 (2005).

4. C. P. Mc Elhinney, J. Maycock, J. B. McDonald, T. J. Naughton, and B. Javidi, "Three-dimensional scene reconstruction using digital holography," in Opto-Ireland 2005: Imaging and Vision, F. D. Murtagh, ed., Proc. SPIE 5823, 48-57 (2005).

5. D. Gabor, "A new microscopic principle," Nature 161, 777-778 (1948).

6. J. W. Goodman and R. W. Lawrence, "Digital image formation from electronically detected holograms," Appl. Phys. Lett. 11, 77-79 (1967).

7. L. P. Yaroslavskii and N. S. Merzlyakov, Methods of Digital Holography (Consultants Bureau, Plenum, 1980).

8. J. H. Bruning, D. R. Herriott, J. E. Gallagher, D. P. Rosenfeld, A. D. White, and D. J. Brangaccio, "Digital wavefront measuring interferometer for testing optical surfaces and lenses," Appl. Opt. 13, 2693-2703 (1974).

9. U. Schnars and W. P. O. Jüptner, "Direct recording of holograms by a CCD target and numerical reconstruction," Appl. Opt. 33, 179-181 (1994).

10. I. Yamaguchi and T. Zhang, "Phase-shifting digital holography," Opt. Lett. 22, 1268-1270 (1997).

11. L. Onural and P. D. Scott, "Digital decoding of in-line holograms," Opt. Eng. 26, 1124-1132 (1987).

12. B. Javidi and E. Tajahuerce, "Three-dimensional object recognition by use of digital holography," Opt. Lett. 25, 610-612 (2000). 
13. Y. Frauel, E. Tajahuerce, M.-A. Castro, and B. Javidi, "Distortion-tolerant three-dimensional object recognition with digital holography," Appl. Opt. 40, 3887-3893 (2001).

14. T. J. Naughton, Y. Frauel, B. Javidi, and E. Tajahuerce, "Compression of digital holograms for three-dimensional object reconstruction and recognition," Appl. Opt. 41, 4124-4132 (2002).

15. T. J. Naughton, J. B. McDonald, and B. Javidi, "Efficient compression of Fresnel fields for Internet transmission of threedimensional images," Appl. Opt. 42, 4758-4764 (2003).

16. T. J. Naughton and B. Javidi, "Compression of encrypted three-dimensional objects using digital holography," Opt. Eng. 43, 2233-2238 (2004).

17. O. Matoba, T. J. Naughton, Y. Frauel, N. Bertaux, and B. Javidi, "Real-time three-dimensional object reconstruction by use of a phase-encoded digital hologram," Appl. Opt. 41, 61876192 (2002).

18. E. Tajahuerce and B. Javidi, "Encrypting three-dimensional information with digital holography," Appl. Opt. 39, 65956601 (2000).

19. T. Nomura, A. Okazaki, M. Kameda, Y. Morimoto, and B. Javidi, "Image reconstruction from compressed encrypted digital hologram," Opt. Eng. 44, 075801 (2005).

20. J. W. Goodman, Introduction to Fourier Optics, 3rd ed., (Roberts \& Company, 2005).

21. H. J. Caulfield, ed., Handbook of Optical Holography (Academic, 1979).

22. E. Wigner, "On the quantum correction for thermodynamic equilibrium," Phys. Rev. 40, 749-759 (1932).

23. M. J. Bastians, "Application of the Wigner distribution function in optics," in The Wigner Distribution-Theory and Applications in Signal Processing, W. Mecklenbrauker and F. Hlawatsch, eds. (Elsevier Science, 1997).

24. A. W. Lohmann, "Image rotation, Wigner rotation and the fractional Fourier transform," J. Opt. Soc. Am. A 10, 21812186 (1993).

25. H. M. Ozaktas, Z. Zalevsky, and M. A. Kutay, The Fractional
Fourier Transform with Applications in Optics and Signal Processing (Wiley, 2001).

26. A. W. Lohmann, R. G. Dorsch, D. Mendolovic, Z. Zalevsky, and C. Ferreira, "Space-bandwidth product of optical signals and systems,” J. Opt. Soc. Am. A 13, 470-473 (1996).

27. A. Stern and B. Javidi, "Sampling in the light of Wigner distribution: eratta," J. Opt. Soc. Am. A 21, 360-366 (2004).

28. A. Stern and B. Javidi, "Sampling in the light of Wigner distribution," J. Opt. Soc. Am. A 21, 2038-2038 (2004).

29. B. M. Hennelly and J. T. Sheridan "Generalizing, optimizing, and inventing numerical algorithms for the fractional Fourier, Fresnel, and linear canonical transforms," J. Opt. Soc. Am. A 21, 917-927 (2005).

30. B. M. Hennelly and J. T. Sheridan "Tracking the space bandwidth product in optical systems," in Opto-Ireland 2005: Photonic Engineering, B. W. Bowe, G. Byrne, A. J. Flanogan, T. G. Glynn, J. Magee, G. M. O'Connor, R. F. O'Dowd, G. D. O'Sullivan, and J. T. Sheridan, eds., 5827, 334-345 (2005).

31. C. J. Román-Moreno and R. Ortega-Martínez “The Wigner function in paraxial optics II. Optical diffraction pattern representation," Mex. Fis. 49, 290-295 (2003).

32. A. Stern and B. Javidi, "General sampling theorem and application to digital holography," in Optical Information Systems II, B. Javidi and D. Psaltis, eds., Proc. SPIE 5557, 110-123 (2004).

33. T. Kreis, Handbook of Holographic Interferometry (WileyVCH, 2005).

34. B. Javidi and D. Kim, "Three-dimensional-object recognition by use of singleexposure on-axis digital holography," Opt. Lett. 30, 236-238 (2005).

35. C. P. McElhinney, J. Maycock, T. J. Naughton, J. B. McDonald, and B. Javidi, "Extraction of three-dimensional shape information from a digital hologram," in Optical Information Systems III, B. Javidi and D. Psaltis, eds., Proc. SPIE 5908, 30-41 (2005). 